\title{
Full result for the three-loop static quark potential
}

\author{
Alexander V. Smirnov \\ Scientific Research Computing Center of Moscow State University, Russia \\ E-mail: asmirnov80@gmail.com
}

\section{Vladimir A. Smirnov*}

Nuclear Physics Institute of Moscow State University, Russia

E-mail: smirnovatheory.sinp.msu.ru

\section{Matthias Steinhauser}

Institut für Theoretische Teilchenphysik, Karlsruhe Institute of Technology, Germany

E-mail: matthias.steinhauserakit.edu

The three-loop corrections to the potential of two heavy quarks are computed. Analytic results for the most complicated master integrals are presented.

RADCOR 2009 - 9th International Symposium on Radiative Corrections (Applications of Quantum Field Theory to Phenomenology),

October 25 - 302009

Ascona, Switzerland

\footnotetext{
* Speaker.
} 


\section{Introduction}

The potential formed by two heavy quarks is among the early applications of Quantum Chromodynamics (QCD). It is an important ingredient in the description of the properties of heavyquark bound states. Among the most prominent applications, which require high-order results of the static potential, are the top quark production cross section close to threshold and the extraction of the bottom quark mass from $\Upsilon$ sum rules (see, e.g., Ref. [1] for a review).

The $n$-loop corrections to the quark anti-quark potential are usually parameterized by the constants $a_{i}$ such that in momentum space it takes the form

$$
V(|\vec{q}|)=-\frac{4 \pi C_{F} \alpha_{s}}{\vec{q}^{2}}\left[1+\frac{\alpha_{s}}{4 \pi} a_{1}+\left(\frac{\alpha_{s}}{4 \pi}\right)^{2} a_{2}+\left(\frac{\alpha_{s}}{4 \pi}\right)^{3}\left(a_{3}+8 \pi^{2} C_{A}^{3} \ln \frac{\mu^{2}}{\vec{q}^{2}}\right)+\cdots\right],
$$

where the renormalization scale has been identified with $|\vec{q}|$ and for $S U\left(N_{c}\right)$ we have $C_{A}=N_{c}$, $C_{F}=\left(N_{c}^{2}-1\right) /\left(2 N_{c}\right)$ and $N_{c}=3$.

The one-loop corrections to $V(|\vec{q}|)$ have been computed more than 30 years ago [2]. Around the same time it has been discovered that $V(|\vec{q}|)$ is not infra-red safe and starting from three-loop order divergences appears [3]. In the 1990ies the two-loop corrections have been computed in the works $[4,5]$ and shortly afterwards the coefficient of the three-loop divergence has been evaluated indirectly by examining the ultra-soft contribution to the energy of two heavy quarks $[6,7]$. First steps towards the finite part of the three-loop corrections have been performed in Refs. [8,9] where the fermionic contributions have been evaluated. The gluonic contribution which completes the knowledge about $a_{3}$ has been obtained by two independent computations [10,11].

The evaluation of Refs. $[9,10]$ is highly automated in order to avoid errors due to manual interactions. After generating the amplitudes for the Feynman diagrams with QGRAF [12] we use q2e and exp [13, 14] in order to rewrite the expressions in FORM [15] format which is used for taking the traces and further simplifications. The reduction to master integrals is performed with the program package FIRE [16] and the resulting master integrals are computed with the help of the Mellin-Barnes technique (see, e.g., Refs. $[17,18,19]$ ). Cross checks are based on the program FIESTA [20] which incorporates the sector decomposition algorithm.

We managed to compute all the necessary coefficients of the $\varepsilon$ expansion of the master integrals analytically with the exception of three terms of order $\varepsilon^{1}$. Results for some master integrals are presented in the next section. Let us close this Section by summarizing the results for $a_{3}$ from Refs. [9, 10]: It is convenient to decompose $a_{3}$ according to the powers of $n_{l}$, the numbers of massless quarks $a_{3}=a_{3}^{(3)} n_{l}^{3}+a_{3}^{(2)} n_{l}^{2}+a_{3}^{(1)} n_{l}+a_{3}^{(0)}$. These are our results:

$$
\begin{aligned}
a_{3}^{(3)}= & -\left(\frac{20}{9}\right)^{3} T_{F}^{3}, \\
a_{3}^{(2)}= & \left(\frac{12541}{243}+\frac{368 \zeta(3)}{3}+\frac{64 \pi^{4}}{135}\right) C_{A} T_{F}^{2}+\left(\frac{14002}{81}-\frac{416 \zeta(3)}{3}\right) C_{F} T_{F}^{2}, \\
a_{3}^{(1)}= & (-709.717) C_{A}^{2} T_{F}+\left(-\frac{71281}{162}+264 \zeta(3)+80 \zeta(5)\right) C_{A} C_{F} T_{F} \\
& +\left(\frac{286}{9}+\frac{296 \zeta(3)}{3}-160 \zeta(5)\right) C_{F}^{2} T_{F}+(-56.83(1)) \frac{d_{F}^{a b c d} d_{F}^{a b c d}}{N_{A}},
\end{aligned}
$$




$$
a_{3}^{(0)}=502.24(1) C_{A}^{3}-136.39(12) \frac{d_{F}^{a b c d} d_{A}^{a b c d}}{N_{A}}
$$

where $T_{F}=1 / 2, d_{F}^{a b c d} d_{F}^{a b c d} / N_{A}=\left(18-6 N_{c}^{2}+N_{c}^{4}\right) /\left(96 N_{c}^{2}\right)$ and $d_{F}^{a b c d} d_{A}^{a b c d} / N_{A}=\left(N_{c}^{3}+6 N_{c}\right) / 48$.

\section{Results for selected master integrals}

If one counts all master integrals for all the types of the integrals appearing in the calculation in the general $\xi$-gauge one obtains around hundred master integrals. However, only 41 master integrals contribute to the three-loop static potential in the Feynman gauge. Eighteen most complicated master integrals are shown in Fig. 1 . Only two of them, $I_{17}$ and $I_{18}$, are non-planar. Diagram $I_{17}$ does not involve static lines and is just a three-loop propagator diagram which can be found in Ref. [21] and which we need up to order $\varepsilon^{1}$. Diagram $I_{18}$ represents the most complicated master integral in our calculation. Explicitly, we have $I_{18}=F_{1, \ldots, 1,0,1,0}^{(n p)}$, where

$$
\begin{aligned}
F_{a_{1}, \ldots, a_{12}}^{(n p)}= & \iiint \frac{\mathrm{d} k \mathrm{~d} l \mathrm{~d} r}{\left(-k^{2}\right)^{a_{1}}\left(-l^{2}\right)^{a_{2}}\left(-r^{2}\right)^{a_{3}}\left(-(r+q)^{2}\right)^{a_{4}}\left(-(k-l+r+q)^{2}\right)^{a_{5}}\left(-(k+q)^{2}\right)^{a_{6}}} \\
& \times \frac{\left(-(k-r)^{2}\right)^{-a_{12}}}{\left(-(l-r)^{2}\right)^{a_{7}}\left(-(k-l)^{2}\right)^{a_{8}}(-v \cdot k)^{a_{9}}(-v \cdot l)^{a_{10}}(-v \cdot r)^{a_{11}}},
\end{aligned}
$$

with the causal $-i 0$ implied in all propagators. It turns out that it is more convenient to use, instead of this master integral, a master integral with a numerator: $I_{18}=F_{1, \ldots, 1,-2,1,0}^{(\mathrm{np})}$. It is finite at $d=4$ and its value is one of three (yet) analytically unknown constants. The other two pieces of the three-loop static potential which are only known numerically are the $O(\varepsilon)$ terms in $I_{11}$ and $I_{16}$.

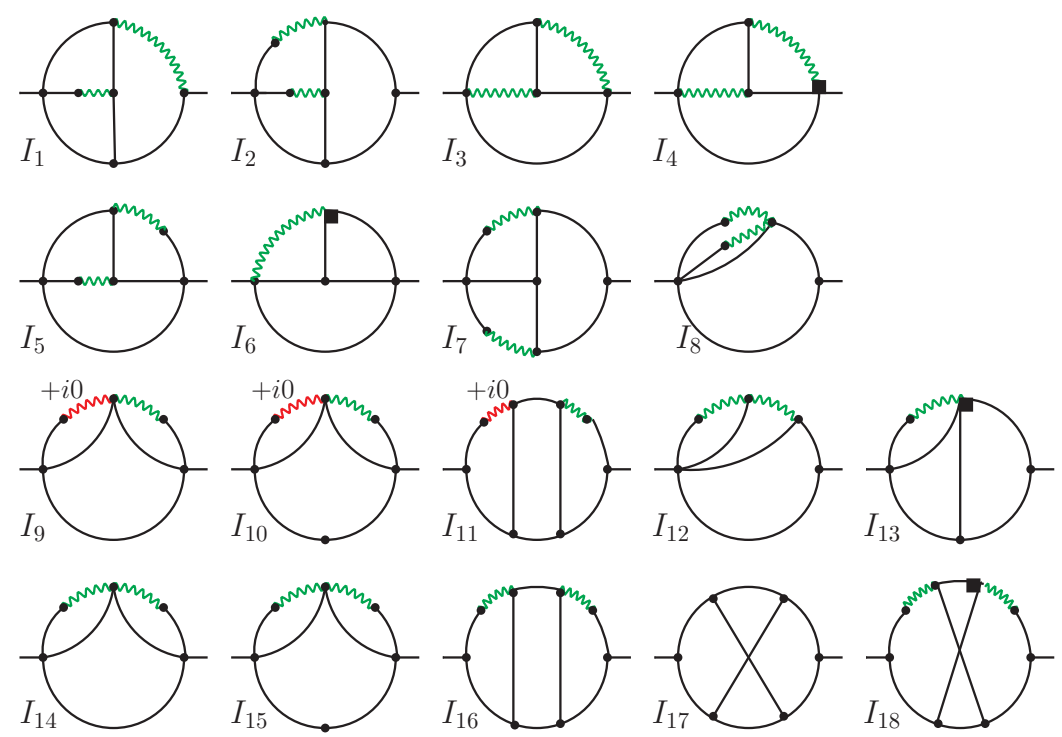

Figure 1: Most complicated master integrals contributing to $a_{3}$. The solid and zig-zag lines correspond to relativistic and static propagators, respectively. Small black boxes denote monomials in numerators. 
In the following we present our analytic results for the 16 most complicated master integrals which are conveniently expressed as special cases of one of the four functions

$$
\begin{aligned}
F_{a_{1}, \ldots, a_{12}}^{(1)}= & \iiint \frac{\mathrm{d} k \mathrm{~d} l \mathrm{~d} r}{\left(-(k+q)^{2}\right)^{a_{1}}\left(-(r+q)^{2}\right)^{a_{2}}\left(-r^{2}\right)^{a_{3}}\left(-l^{2}\right)^{a_{4}}\left(-k^{2}\right)^{a_{5}}\left(-(k-r)^{2}\right)^{a_{6}}} \\
& \times \frac{\left(-(l+q)^{2}\right)^{a_{12}}}{\left(-(l-r)^{2}\right)^{a_{7}}\left(-(k-l)^{2}\right)^{a_{8}}(-v \cdot k)^{a_{9}}(-v \cdot r)^{a_{10}}(-v \cdot(k-l))^{a_{11}}}, \\
F_{a_{1}, \ldots, a_{12}}^{(2)}= & \iiint \frac{\mathrm{d} k \mathrm{~d} l \mathrm{~d} r}{\left(-(k+q)^{2}\right)^{a_{1}}\left(-(r+q)^{2}\right)^{a_{2}}\left(-r^{2}\right)^{a_{3}}\left(-l^{2}\right)^{a_{4}}\left(-k^{2}\right)^{a_{5}}} \\
& \times \frac{\left(-(l+q)^{2}\right)^{a_{12}}}{\left(-(k-r)^{2}\right)^{a_{6}}\left(-(l-r)^{2}\right)^{a_{7}}\left(-(k-l)^{2}\right)^{a_{8}}(-v \cdot k)^{a_{9}}(-v \cdot r)^{a_{10}}(-v \cdot l)^{a_{11}}}, \\
F_{a_{1}, \ldots, a_{12}=}^{(3)}= & \iiint \frac{\mathrm{d} k \mathrm{~d} l \mathrm{~d} r}{\left(-k^{2}\right)^{a_{1}}\left(-l^{2}\right)^{a_{2}}\left(-r^{2}\right)^{a_{3}}\left(-(r+q)^{2}\right)^{a_{4}}\left(-(l+q)^{2}\right)^{a_{5}}\left(-(k+q)^{2}\right)^{a_{6}}} \\
& \times \frac{\left(-(k-r)^{2}\right)^{-a_{12}}}{\left(-(k-l)^{2}\right)^{a_{7}}\left(-(l-r)^{2}\right)^{a_{8}}(-v \cdot k)^{a_{9}}(-v \cdot(k-l))^{a_{10}}(-v \cdot r)^{a_{11}}}, \\
F_{a_{1}, \ldots, a_{12}}^{(4, \pm)}= & \iiint \frac{\mathrm{d} k \mathrm{~d} l \mathrm{~d} r}{\left(-k^{2}\right)^{a_{1}}\left(-l^{2}\right)^{a_{2}}\left(-r^{2}\right)^{a_{3}}\left(-(r+q)^{2}\right)^{a_{4}}\left(-(l+q)^{2}\right)^{a_{5}}\left(-(k+q)^{2}\right)^{a_{6}}} \\
& \times \frac{\left(-(k-r)^{2}\right)^{-a_{12}}}{\left(-(k-l)^{2}\right)^{a_{7}}\left(-(l-r)^{2}\right)^{a_{8}}(-v \cdot k \mp i 0)^{a_{9}}(-v \cdot l)^{a_{10}}(-v \cdot r)^{a_{11}}},
\end{aligned}
$$

where in all propagators, apart \#9, the causal $-i 0$ is implied. Here are our results:

$$
\begin{gathered}
I_{1}=F_{1,0,1,1,0,1,1,1,0,1,1,0}^{(1)}=\frac{1}{Q^{2} v^{2}}\left[-\frac{28 \pi^{4}}{135 \varepsilon}+\frac{188 \zeta(5)}{3}-\frac{152 \pi^{2} \zeta(3)}{9}+\frac{112 \pi^{4}}{135}+O(\varepsilon)\right] \\
I_{2}=F_{1,1,1,1,0,1,1,1,1,0,1,0}^{(1)}=\frac{1}{Q^{4} v^{2}}\left[-\frac{44 \pi^{4}}{135 \varepsilon}-\frac{92 \zeta(5)}{3}-\frac{64 \pi^{2} \zeta(3)}{9}-\frac{88 \pi^{4}}{135}+O(\varepsilon)\right] \\
I_{3}=F_{1,0,0,1,0,1,1,0,0,1,1,0}^{(1)}=\frac{\pi^{2} Q^{2}}{v^{2}}\left[-\frac{1}{9 \varepsilon}+\frac{4 \log (2)}{9}-\frac{41}{27}\right. \\
\quad+\left(-\frac{1201}{81}+\frac{43 \pi^{2}}{108}+\frac{92 \log (2)}{27}+\frac{8 \log ^{2}(2)}{9}\right) \varepsilon+\left(-\frac{31289}{243}+\frac{1475 \pi^{2}}{324}\right. \\
\left.\left.\quad+\frac{988 \log (2)}{81}-\frac{11}{27} \pi^{2} \log (2)+\frac{184 \log ^{2}(2)}{27}+\frac{32 \log ^{3}(2)}{27}+\frac{85 \zeta(3)}{3}\right) \varepsilon^{2}+O\left(\varepsilon^{3}\right)\right] \\
I_{4}=F_{1,-1,0,1,0,1,1,0,0,1,1,0}^{(1)}=\frac{\pi^{2} Q^{4}}{v^{2}}\left[-\frac{7}{360 \varepsilon}+\frac{\log ^{2}(2)}{10}-\frac{3041}{10800}\right. \\
+\left(-\frac{909193}{324000}+\frac{37 \pi^{2}}{480}+\frac{2047 \log (2)}{2700}+\frac{\log ^{2}(2)}{5}\right) \varepsilon+\left(-\frac{238569389}{9720000}+\frac{114859 \pi^{2}}{129600}\right. \\
\left.\left.+\frac{78077 \log (2)}{27000}-\frac{11}{120} \pi^{2} \log (2)+\frac{2047 \log ^{2}(2)}{1350}+\frac{4 \log ^{3}(2)}{15}+\frac{389 \zeta(3)}{72}\right) \varepsilon^{2}+O(\varepsilon)^{3}\right] \\
I_{5}=F_{1,1,0,1,0,1,1,1,0,1,1,0}^{(1)}=\frac{1}{Q^{2} v^{2}}\left[-\frac{32 \pi^{4}}{135 \varepsilon}-\frac{188 \zeta(5)}{3}-\frac{88 \pi^{2} \zeta(3)}{9}+\frac{128 \pi^{4}}{135}+O(\varepsilon)\right] \\
I_{6}=F_{0,1,0,1,0,1,1,1,1,-1,0,0}^{(2)}=Q^{2}\left[\left(-\frac{5}{72}-\frac{\zeta(3)}{6}\right) \frac{1}{\varepsilon}-\frac{13 \zeta(3)}{9}-\frac{437}{432}\right. \\
\left.+\left(-\frac{23471}{2592}+\frac{5 \pi^{2}}{288}-\frac{293 \zeta(3)}{27}+\frac{\pi^{2} \zeta(3)}{24}+\frac{19 \zeta(5)}{6}\right) \varepsilon+O\left(\varepsilon^{2}\right)\right]
\end{gathered}
$$




$$
\begin{aligned}
& I_{7}=F_{1,1,1,1,0,1,1,1,1,0,1,0}^{(2)}=\frac{1}{Q^{4} v^{2}}\left[-\frac{32 \pi^{4}}{135 \varepsilon}+\frac{184 \zeta(5)}{3}-\frac{64 \pi^{2} \zeta(3)}{9}-\frac{64 \pi^{4}}{135}+O(\varepsilon)\right], \\
& I_{8}=F_{1,0,1,1,0,0,1,1,1,1,0,0}^{(3)}=\frac{1}{v^{2}}\left[\frac{2 \pi^{2}}{9 \varepsilon^{2}}+\left(\frac{16 \pi^{2}}{9}+\frac{8 \zeta(3)}{3}\right) \frac{1}{\varepsilon}+\frac{112 \zeta(3)}{3}+\frac{61 \pi^{4}}{270}+\frac{56 \pi^{2}}{9}\right. \\
& +\left(-\frac{416 \pi^{2}}{9}+\frac{628 \pi^{4}}{135}+\frac{1184 \zeta(3)}{3}+\frac{152 \pi^{2} \zeta(3)}{9}-\frac{232 \zeta(5)}{3}\right) \varepsilon+\left(-\frac{11104 \pi^{2}}{9}+\frac{8534 \pi^{4}}{135}\right. \\
& \left.\left.+\frac{77647 \pi^{6}}{45360}+\frac{11200 \zeta(3)}{3}+\frac{2908 \pi^{2} \zeta(3)}{9}-\frac{1240 \zeta(3)^{2}}{3}-\frac{2864 \zeta(5)}{3}\right) \varepsilon^{2}+O\left(\varepsilon^{3}\right)\right], \\
& I_{9}=F_{1,0,1,0,1,0,1,1,1,0,1,0}^{(4,-)}=\frac{1}{v^{2}}\left[-\frac{32 \pi^{4}}{135 \varepsilon}+\frac{226 \zeta(5)}{3}-\frac{232 \pi^{2} \zeta(3)}{9}+4 \pi^{4} \log (2)-\frac{256 \pi^{4}}{135}\right. \\
& +\left(-192 s_{6}+\frac{1808 \zeta(5)}{3}-\frac{8 \zeta(3)^{2}}{3}-\frac{1856 \pi^{2} \zeta(3)}{9}-128 \pi^{2} \operatorname{Li}_{4}\left(\frac{1}{2}\right)-\frac{16}{3} \pi^{2} \log ^{4}(2)\right. \\
& \left.\left.+\frac{28}{3} \pi^{4} \log ^{2}(2)+32 \pi^{4} \log (2)+\frac{2344 \pi^{6}}{2835}-\frac{2048 \pi^{4}}{135}\right) \varepsilon+O\left(\varepsilon^{2}\right)\right], \\
& I_{10}=F_{1,0,1,0,2,0,1,1,1,0,1,0}^{(4,-)}=\frac{1}{Q^{2} v^{2}}\left[\frac{\pi^{4}}{\varepsilon}-93 \zeta(5)+28 \pi^{2} \zeta(3)+2 \pi^{4} \log (2)\right. \\
& \left.+\left(-96 s_{6}+120 \zeta(3)^{2}-64 \pi^{2} \operatorname{Li}_{4}\left(\frac{1}{2}\right)-\frac{8}{3} \pi^{2} \log ^{4}(2)+\frac{14}{3} \pi^{4} \log ^{2}(2)+\frac{887 \pi^{6}}{420}\right) \varepsilon+O\left(\varepsilon^{2}\right)\right], \\
& I_{11}=F_{1,1,1,1,1,1,1,1,1,0,1,0}^{(4,-)}=\frac{1}{Q^{6} v^{2}}\left[\frac{64 \pi^{4}}{135 \varepsilon}-\frac{8 \zeta(5)}{3}+\frac{32 \pi^{2} \zeta(3)}{9}+\frac{128 \pi^{4}}{135}+O(\varepsilon)\right] \\
& I_{12}=F_{1,0,1,1,0,0,1,1,1,1,0,0}^{(4,+)}=\frac{1}{Q^{6} v^{2}}\left[\frac{2 \pi^{2}}{9 \varepsilon^{2}}-\frac{4 \zeta(3)}{3 \varepsilon}+\frac{16 \pi^{2}}{9 \varepsilon}-\frac{56 \zeta(3)}{3}+\frac{157 \pi^{4}}{270}+\frac{56 \pi^{2}}{9}\right. \\
& +\left(-\frac{416 \pi^{2}}{9}+\frac{1156 \pi^{4}}{135}-\frac{592 \zeta(3)}{3}+\frac{161 \pi^{2} \zeta(3)}{9}+\frac{116 \zeta(5)}{3}\right) \varepsilon+\left(-\frac{11104 \pi^{2}}{9}+\frac{12758 \pi^{4}}{135}\right. \\
& \left.\left.+\frac{18563 \pi^{6}}{9072}-\frac{5600 \zeta(3)}{3}+\frac{3034 \pi^{2} \zeta(3)}{9}+\frac{620 \zeta(3)^{2}}{3}+\frac{1432 \zeta(5)}{3}\right) \varepsilon^{2}+O\left(\varepsilon^{3}\right)\right], \\
& I_{13}=F_{1,0,1,1,1,0,1,1,1,-1,0,0}^{(4,+)}=\frac{1}{6 \varepsilon^{2}}+\frac{3}{2 \varepsilon}-18 \zeta(5)+18 \zeta(3)-\frac{\pi^{2}}{24}+\frac{25}{6} \\
& +\left(-\frac{105}{2}-\frac{3 \pi^{2}}{8}+\frac{3 \pi^{4}}{5}-\frac{2 \pi^{6}}{63}+\frac{619 \zeta(3)}{6}-50 \zeta(3)^{2}\right) \varepsilon+O\left(\varepsilon^{2}\right), \\
& I_{14}=F_{1,0,1,0,1,0,1,1,1,0,1,0}^{(4,+)}=\frac{1}{v^{2}}\left[\frac{28 \pi^{4}}{135 \varepsilon}+\frac{116 \pi^{2} \zeta(3)}{9}+\pi^{4}\left(\frac{224}{135}-4 \log (2)\right)+\frac{226 \zeta(5)}{3}\right. \\
& +\left(-192 s_{6}+\frac{1808 \zeta(5)}{3}-\frac{8 \zeta(3)^{2}}{3}+\frac{928 \pi^{2} \zeta(3)}{9}+64 \pi^{2} \operatorname{Li}_{4}\left(\frac{1}{2}\right)+\frac{8}{3} \pi^{2} \log ^{4}(2)\right. \\
& \left.\left.-\frac{20}{3} \pi^{4} \log ^{2}(2)-32 \pi^{4} \log (2)-\frac{428 \pi^{6}}{2835}+\frac{1792 \pi^{4}}{135}\right) \varepsilon+O\left(\varepsilon^{2}\right)\right], \\
& I_{15}=F_{1,0,1,0,2,0,1,1,1,0,1,0}^{(4,+)}=\frac{1}{Q^{2} v^{2}}\left[-\frac{\pi^{4}}{\varepsilon}-93 \zeta(5)-14 \pi^{2} \zeta(3)-2 \pi^{4} \log (2)\right. \\
& \left.+\left(-96 s_{6}+120 \zeta(3)^{2}+32 \pi^{2} \operatorname{Li}_{4}\left(\frac{1}{2}\right)+\frac{4}{3} \pi^{2} \log ^{4}(2)-\frac{10}{3} \pi^{4} \log ^{2}(2)-\frac{989 \pi^{6}}{420}\right) \varepsilon+O\left(\varepsilon^{2}\right)\right],
\end{aligned}
$$




$$
I_{16}=F_{1,1,1,1,1,1,1,1,1,0,1,0}^{(4,+)}=\frac{1}{Q^{6} v^{2}}\left[-\frac{56 \pi^{4}}{135 \varepsilon}-\frac{8 \zeta(5)}{3}-\frac{16 \pi^{2} \zeta(3)}{9}-\frac{112 \pi^{4}}{135}+O(\varepsilon)\right]
$$

where $Q=\sqrt{-q^{2}}$ and $\left(i \pi^{d / 2} e^{-\gamma_{E} \varepsilon}\right)^{3}$ is implied as a factor on the right-hand side. These $\varepsilon$ expansions are up to the order which contributes to the static potential, with the exception of $I_{11}$ and $I_{16}$, where one more order is desirable. Results for all the master integrals as well as details of their calculation will be published elsewhere.

Acknowledgements. This work is supported by DFG through project SFB/TR 9 and RFBR, grant 08-02-01451. V.S. appreciates the financial support of the organizers of the Symposium.

\section{References}

[1] N. Brambilla et al. [Quarkonium Working Group], arXiv:hep-ph/0412158.

[2] W. Fischler, Nucl. Phys. B 129 (1977) 157; A. Billoire, Phys. Lett. B 92 (1980) 343.

[3] T. Appelquist, M. Dine and I. J. Muzinich, Phys. Rev. D 17 (1978) 2074.

[4] M. Peter, Phys. Rev. Lett. 78 (1997) 602 [arXiv:hep-ph/9610209]; Nucl. Phys. B 501 (1997) 471 [arXiv:hep-ph/9702245].

[5] Y. Schroder, Phys. Lett. B 447 (1999) 321 [arXiv:hep-ph/9812205].

[6] N. Brambilla, A. Pineda, J. Soto and A. Vairo, Phys. Rev. D 60 (1999) 091502 [arXiv:hep-ph/9903355].

[7] B. A. Kniehl, A. A. Penin, V. A. Smirnov and M. Steinhauser, Nucl. Phys. B 635 (2002) 357 [arXiv:hep-ph/0203166].

[8] A. V. Smirnov, V. A. Smirnov and M. Steinhauser, PoS RADCOR2007 (2007) 024 [arXiv:0805.1871]; Nucl. Phys. Proc. Suppl. 183 (2008) 308 [arXiv:0807.0365].

[9] A. V. Smirnov, V. A. Smirnov and M. Steinhauser, Phys. Lett. B 668 (2008) 293 [arXiv:0809.1927].

[10] A. V. Smirnov, V. A. Smirnov and M. Steinhauser, arXiv:0911.4742.

[11] C. Anzai, Y. Kiyo and Y. Sumino, arXiv:0911.4335.

[12] P. Nogueira, J. Comput. Phys. 105 (1993) 279.

[13] R. Harlander, T. Seidensticker and M. Steinhauser, Phys. Lett. B 426 (1998) 125 [hep-ph/9712228].

[14] T. Seidensticker, hep-ph/9905298.

[15] J. A. M. Vermaseren, arXiv:math-ph/0010025.

[16] A. V. Smirnov, arXiv:0807.3243.

[17] V. A. Smirnov, "Evaluating Feynman Integrals," Springer Tracts Mod. Phys. 211 (2004) 1; “Feynman integral calculus," Berlin, Germany: Springer (2006) 283 p.

[18] M. Czakon, Comput. Phys. Commun. 175 (2006) 559 [arXiv:hep-ph/0511200].

[19] A.V. Smirnov and V. A. Smirnov, Eur. Phys. J. C 62 (2009) 445 [arXiv:0901.0386].

[20] A. V. Smirnov and M. N. Tentyukov, Comput. Phys. Commun. 180 (2009) 735 [arXiv:0807.4129]; A. V. Smirnov, V. A. Smirnov and M. Tentyukov, arXiv:0912.0158.

[21] K. G. Chetyrkin, A. L. Kataev and F. V. Tkachov, Nucl. Phys. B 174 (1980) 345; D. I. Kazakov, Teor. Mat. Fiz. 58 (1984) 343; S. Bekavac, Comput. Phys. Commun. 175 (2006) 180 [arXiv:hep-ph/0505174]. 